NBER WORKING PAPER SERIES

\title{
RETURNS TO PHARMACEUTICAL INNOVATION IN THE MARKET FOR ORAL CHEMOTHERAPY IN RESPONSE TO INSURANCE COVERAGE EXPANSION
}

\author{
Caroline S. Bennette \\ Anirban Basu \\ Scott D. Ramsey \\ Zachary Helms \\ Peter B. Bach \\ Working Paper 23842 \\ http://www.nber.org/papers/w23842 \\ NATIONAL BUREAU OF ECONOMIC RESEARCH \\ 1050 Massachusetts Avenue \\ Cambridge, MA 02138 \\ September 2017
}

Caroline Bennette was supported by an institutional mentored career development award from the Agency for Healthcare Research and Quality (PI: Sean Sullivan; No. K12HS022982). Dr. Bennette was also supported by a grant from the Laura and John Arnold Foundation (PI: Peter Bach). We are grateful for comments on earlier versions of this work from seminar participants at the University of Washington and the Vancouver Health Economics Meeting. Opinions expressed are the authors' alone and do not reflect those of the University of Washington, NBER, Flatiron Health, the Agency for Healthcare Research and Quality or the Memorial Sloan Kettering Cancer Center. NBER working papers are circulated for discussion and comment purposes. They have not been peer reviewed or been subject to the review by the NBER Board of Directors that accompanies official NBER publications. The views expressed herein are those of the authors and do not necessarily reflect the views of the National Bureau of Economic Research.

NBER working papers are circulated for discussion and comment purposes. They have not been peer-reviewed or been subject to the review by the NBER Board of Directors that accompanies official NBER publications.

(C) 2017 by Caroline S. Bennette, Anirban Basu, Scott D. Ramsey, Zachary Helms, and Peter B. Bach. All rights reserved. Short sections of text, not to exceed two paragraphs, may be quoted without explicit permission provided that full credit, including $\odot$ notice, is given to the source. 
Returns to Pharmaceutical Innovation in the Market for Oral Chemotherapy in Response to Insurance Coverage Expansion

Caroline S. Bennette, Anirban Basu, Scott D. Ramsey, Zachary Helms, and Peter B. Bach

NBER Working Paper No. 23842

September 2017

JEL No. I11,I13,I18

\section{ABSTRACT}

We estimated the average returns, in terms of patient survival, to the marginal innovations in oral chemotherapy market induced by Part D expansion of oral chemotherapy coverage for elderly individuals by mandating inclusion of "all or substantially all" oral anti-cancer medications on plans' formularies. We exploited exogenous variation in the age of diagnosis for different cancer sites - and therefore the relative expansion in market size for different cancers under the Medicare's prescription drug coverage - to isolate the effect of Part D on innovation and the health benefits that these innovative technologies provide. Using data from FDA and clinical studies from January 1994 to December 2016, we find that the approval rate for oral chemotherapies increased an additional 5.7\% (95\% CI: 1.7, 9.8) after implementation of Part D for every $1 \%$ increase in exposure to the Medicare market. In contrast, greater exposure to Medicare was associated with a smaller increase in the indication-specific survival gains reported in the drug's label (3.2\% [95\% CI: 2.1, 4.3]) and 8.0\% [95\% CI: 6.1, 9.8] lower in absolute and relative gains, respectively). Similar trends were not observed for intravenously administered chemotherapy whose coverage was largely unaffected by Part D. These findings suggest that there could be diminishing returns to incentives for pharmaceutical innovation created by broad coverage mandates and that health policy tools, such as value-based pricing, may help maximize the health benefits provided by future pharmaceutical innovations.

Caroline S. Bennette

Flatiron Health

New York, NY 10010

cbennette@flatiron.com

Anirban Basu

Departments of Health Services, Pharmacy and Economics

University of Washington

1959 NE Pacific St

Box - 357660

Seattle WA 98195

and NBER

basua@uw.edu

Scott D. Ramsey

Fred Hutchinson Cancer Research Center

1100 Fairview Ave. North, M3-B232

Seattle, WA 98109-1024

sramsey@fhcrc.org
Zachary Helms

Center for Health Policy and Outcomes

Memorial Sloan Kettering Cancer Center

zachary.t.helms@gmail.com

Peter B. Bach

Department of Epidemiology and Biostatistics

Department of Medicine

Memorial Sloan Kettering Cancer Center

New York, NY 10065

bachp@mskcc.org 


\section{Introduction}

The high and rising cost of many prescription drugs is placing tremendous financial pressure on patients, payers, and society. These trends have prompted considerable debate about how best to foster pharmaceutical innovation while ensuring patient access to affordable medications (HHS Pharmaceutical Forum; Ramsey et al. 2016; Mailankody and Prasad 2016; Bennette et al. 2016 ). Strengthening insurance coverage, such as through government subsidies or mandated benefits, is an often-used lever through which policymakers can improve patients' access to prescription drugs (Morgan and Kennedy 2010). Importantly, expanding prescription drug insurance coverage can also alter incentives for pharmaceutical firms' research and development (R\&D) investments and ultimately access to new medicines for future generations (Acemoglu and Linn 2004; Blume-Kohout and Sood 2013).

Prior research has shown that changes in insurance coverage can have a significant impact on pharmaceutical innovation (Acemoglu and Linn 2004; Blume-Kohout and Sood 2013). However, these studies relied on definitions of innovation - such as the number of new drugs developed - that do not capture the health benefits of such innovation (Morgan et al. 2008). Even drugs designed as "breakthrough" therapies by the FDA exhibit large variation in the expected health benefits offered to patients. This variation leaves policymakers and other decision makers with little direct evidence about how policies that impact pharmaceutical innovation will ultimately impact population health (Downing et al. 2014; Kim et al. 2015; Prasad et al. 2015). It is critical to understand how changes in prescription-drug-insurance coverage impact not only the number but also the health benefits provided by drugs ultimately brought to market if we are to design insurance models and policies in a manner that ensures the greatest expected health benefits for both current and future patients.

The need to refine and develop new insurance payment models and coverage policies is particularly acute in oncology given cancer's large human and economic burden and the rapidly growing output of new drugs to treat cancer from the pharmaceutical industry. Anti-cancer medications are one of the three main pillars of cancer treatment 
and recently became the most expensive therapeutic class of drugs. These trends are fueled by a recent surge in the development and FDA approval of new therapies, particularly orally administered chemotherapy (Martell et all. 2013). These new drugs can deliver substantial health benefits to patients, but are often extraordinarily expensive: the costs of chemotherapy now routinely exceed $\$ 10,000$ per month (Bach 2009). In response to these trends, a series of Institute of Medicine workshops discussed the need to ensure all people with cancer have access to affordable anti-cancer drugs (IOM 2013; 2014); however, many questions remain about how such goals should be achieved.

To inform this ongoing discussion, we evaluated how the implementation of Medicare Part D - the largest expansion of prescription drug coverage in United States' history - impacted the development oral anti-cancer medications. Medicare Part D was signed into law on December 8, 2003, by President George W. Bush. Before implementation of Part D legislation on 1 January 2006, only intravenously administered chemotherapy was covered under Medicare through the Part B (i.e. medical insurance) program. Although the legislation creating Part D prohibited the federal government from establishing a national formulary, Part D regulations require all health plans to cover "all or substantially all" drugs in six protected classes, which include anti-cancer medications, to help ensure Medicare beneficiaries' access to these treatments.

Because the average age of diagnosis of cancer vary across different cancer sites, the passage of Medicare Part D created exogenous variation to the relative size of expansion for the insured market of oncology drugs across cancer sites. We exploit this variation to study how expansion of insurance coverage affect innovation and, importantly, how the average returns from these marginal innovations compare to their predecessors.

We hypothesized that the Part D expansion of prescription drug insurance and mandated coverage of all oral chemotherapy agents would fuel pharmaceutical R\&D and ultimately lead to a significant increase in the number of oral chemotherapy agents approved by the FDA via two mechanisms. First, prior studies have shown that firms' R\&D investments increase proportionally to their revenues from sales, which increased substantially upon implementation of Part D (Scherer 2001; Lakdawalla et al. 2013). 
Second, economic theory and prior research indicate that pharmaceutical firms' R\&D investments are responsive to changes in expected profitability of the products in their pipeline (Blume-Kohout and Sood 2013; Friedman 2009).

We further hypothesized that the marginal oral chemotherapy agents approved would be more likely to result from incremental innovation (e.g. "me-too" drugs) and ultimately provide diminishing returns to health. This hypothesis stems from several reasons. First, the short duration over which innovators try to bring innovations to market in response the coverage incentive is likely to make innovations use the same technology (e.g., molecular targets) as their predecessors and hence subject to the diminishing returns of technology. Note that some have suggested that the development of me-too drugs within a class is better characterized as a race, rather than the imitation of successful products (DiMasi and Faden 2011), and hence there is no reason to believe that later entrants would necessary have smaller effects than their predecessors do. While this may be true on average, for me-too drugs marketed in response to exogenous insurance incentive might reflect those that were faring less well in the race and wouldn't have seen the market otherwise, and therefore one can expect diminishing rertuns. Furthermore, because Medicare Part D's coverage mandates provide strong incentives to develop new oral chemotherapy agents, but do not reward the incremental clinical benefits these drugs provide over existing therapies, innovators have the incentive to bring to market innovations with smaller health benefits. Indeed, the coverage mandates require that all oral chemotherapy drugs be included in all Part $D$ formularies regardless of their price or performance relative to alternative treatments (Bowman et al. 2006).

We repeat our analyses for innovations in the intravenous space for oncology, as a falsification test, since Medicare coverage expansion did not affect these products.

We discuss the implications of these hypotheses, and the corresponding results for future public policies in this field in the Discussion Section. 


\section{Methods}

Our overall strategy was to isolate quasi-experimental variation in the incentives created by the Part D program for pharmaceutical firms' to develop new oral chemotherapy drugs by leveraging the fact that cancer is not a single disease, but rather a heterogeneous set of clinical conditions that tend to affect individuals at different ages. We then used time-series data to evaluate the impact of this quasi-experimental variation on the number of oncology drugs approved by the FDA and the incremental health benefits provided by these drugs.

\section{Study Sample}

We assembled data on systemic anti-cancer medications approved by the FDA between January 1994 and December 2016. The dataset included the date(s) each drug was approved, the clinical indication(s), and route of administration (oral or injection/infusion; topically-administered drugs were excluded). We included initial and supplemental FDA approvals for anti-cancer medications used with the intent of extending survival, but excluded those used for chemoprevention or to alleviate side effects of cancer treatment. Our final dataset included 230 FDA approvals for anti-cancer medications.

We used a previously published list of survival benefits associated with the initial FDA approval of anti-cancer drugs approved between 1995 and 2013 and followed a similar approach to estimate the survival benefits for supplemental approvals in new indications and for anti-cancer drugs approved in 1994 and 2014-2016 (Howard et al. 2015). Following the approach used by Howard et al. (2015), we calculated the incremental survival benefit for each approved indication using the improvement in median survival reported by the randomized clinical trial(s) included in the drug's label. We measured benefits by subtracting the median overall survival in the control arm from the median overall survival in the treatment arm; when estimates of overall survival were not reported, we used estimates of progression-free survival. If results from more than one trial were included in the label, we used a weighted average of the survival benefit estimates from both studies (using only the results from the largest or most favorable trial made little difference to our 
findings). If a trial included more than two treatment arms (e.g. multiple doses of an experimental treatment versus a control), we estimated survival benefits as those most favorable to the experimental drug. We did not estimate survival benefits for approvals based on non-survival endpoints (e.g. objective response rate) or single arm studies. Our dataset therefore included estimates of survival benefits for 130 approvals.

\section{Analytic Approach}

We addressed the challenge of isolating the effect of Medicare Part D's coverage expansion on the development of new oral chemotherapy agents amidst tremendous advances in basic science by exploiting quasi-experimental variation in the proportion of patients with different types of cancer who are Medicare-eligible at the time of their diagnosis. We hypothesized that Part D would induce more innovation in oral chemotherapy for cancer sites with a higher proportion of patients who are Medicareeligible. We defined the proportion of each cancer that was Medicare-eligible as the proportion of patients who were $\geq 65$ at the time of diagnosis, which we obtained from the Surveillance, Epidemiology and End Results (SEER) program database (Gold et al. 2013). Our approach rests on the intuition that the variation in age at diagnosis is not caused by the presence of anti-cancer drugs, but it could cause differences in how developing them is prioritized by the biopharmaceutical industry if guaranteed Medicare coverage creates an incentive.

We also hypothesized that the incremental survival benefit of oral chemotherapy agents developed in response to Part D would provide diminishing returns to health. Therapies for cancers that have higher incidence rates in older individuals may provide fewer survival benefits than those developed for cancers that afflict younger individuals simply because younger patients have more years of life left to gain with an effective treatment. Our fixed effects analytic framework leverages changes over time within a cancer site, rather than comparing survival outcomes across cancers, to address this issue. We also repeated our analyses using the relative incremental survival benefit of oral chemotherapy (i.e., the hazard ratio) as our outcome measure. 
Lastly, we repeated all of our analyses using infused chemotherapy agents as a prespecified falsification test, in which the absence of effects is expected (Prasad and Jena 2013). Medicare pays for infused chemotherapy through the Part B outpatient medical benefit. Coverage for infused agents was therefore similar before and after the creation of Part D. A finding of no relationship between Part $D$ and the number and survival benefits of infused chemotherapy agents further strengthens the evidence that the estimated effects in the market for oral chemotherapy are associated with the Part D coverage mandates and not potential unobserved confounders.

\section{Statistical Analyses}

We used fixed effects Poisson regression with robust standard errors to evaluate how introduction of Medicare Part D was associated with changes in: (i) the number of anticancer drugs approved by the FDA each year and (ii) the average number of months these drugs improved median survival (as reported in the FDA label). Cancer sites were entered as fixed effects to allow estimation of the within-cancer changes over time in each outcome and thereby eliminate time-invariant differences in observed and unobserved characteristics between different cancers that are potentially correlated with our outcome measures. We also included year fixed effects to absorb differences across time in unobserved characteristics that are common to all cancer sites and included a dummy variable for the pre- versus post-Part D time period. We adjusted for the annual incidence of each cancer over time to capture differential changes in the market for different cancers that might impact pharmaceutical R\&D investments. Finally, we included an interaction term between the proportion of each cancer site that was Medicare-eligible and the postPart $D$ time period. The coefficient from these interaction terms, which can be thought of as a ratio of the outcome measures in the pre versus post-Part $D$ time periods, would be greater than one if cancer sites that have a greater relative exposure to the Medicare market experienced larger increases in the outcome of interest.

Our model specification is as follows: 


$$
\begin{aligned}
& E\left(y_{j t} \mid .\right)=\exp \left(\left(\beta_{0}+v_{j}\right)+\beta_{1} \text { Post }_{t}+\beta_{2} \text { Share }_{j} * \text { Post }_{t}+\beta X_{j t}+\omega_{t}\right) \text {, where } \\
& v_{j}=\text { cancer-specific fixed effects } \\
& \omega_{t}=\text { year-specific fixed effects } \\
& \text { Post }_{t}=\text { indicator for Post-Medicare Part D period } \\
& \text { Share }_{j}=\text { Cancer-site specific share of Medicare eligibility } \\
& X_{j t}=\text { Annual incidence of each cancer over time }
\end{aligned}
$$

The clinical testing and approval process for new drugs is estimated to take approximately 4-12 years (Blume-Kohout and Sood 2013). In our main analyses, we therefore compared rates of our outcomes in the ten years before Medicare Part D was enacted (January 1994-December 2003) to the 4-12 years after it was enacted (January 2008-December 2016). We hypothesized stronger effects over time after Part D was enacted and conducted sensitivity analyses to examine such trends. All analyses were conducted in STATA 14 (Stata Corp, College Station, TX).

\section{Results}

The rate oral and infused chemotherapy agents approved by the FDA increased substantially from the pre-Part D (1994-2003) to post-Part D (2008-2016) time periods; however, there were important differences between oral and infused agents and across cancer sites. The relative change in the number of oral chemotherapy agents approved for particular cancers was strongly associated with the proportion of individuals with that cancer who were Medicare-eligible (Figure 1a; $\mathrm{p}=0.004$ ). We found a much smaller and non-significant trend for infused chemotherapy (Figure 1b; $p=0.23$ ).

\section{FDA approval of oral chemotherapy agents}

In multivariable fixed effects regression analyses, we found that greater exposure to the Medicare market was associated with a greater increase in the number of oral chemotherapy agents approved after Medicare Part D was implemented (Table 1). The rate oral anti-cancer drugs were approved by the FDA increased an additional 5.7\% (95\% Cl: 
$1.7,9.8)$ after Part $D$ was implemented for every $1 \%$ increase in exposure to the Medicare market. We also evaluated the timing of these associations and found no evidence that the relationship between the level of exposure to the Medicare market and oral chemotherapy FDA approvals preceded Part $D$, but observed an increasingly robust trend in the time periods post-Part D (Figure 2a).

\section{Survival benefits of oral chemotherapy agents}

Greater exposure to the Medicare market was associated with a smaller increase in the improvement in median survival reported in the FDA label for each newly approved oral chemotherapy indication. The absolute improvement in median survival associated with approvals after Part D was 3.2 percent (95\% Cl: 2.1, 4.3; $p<0.001$; Table 2) lower for every $1 \%$ increase in exposure to the Medicare market. The effect of Part $D$ on the relative improvement in survival (i.e., the hazard ratio scale) with these drugs was an 8.0 percent (95\% Cl: 6.1, 9.8; $p<0.001)$ decline in effectiveness for every $1 \%$ increase in exposure to the Medicare market. To translate these results to returns to innovation, we calculated an elasticity of incremental absolute survival benefit with respect to pharmaceutical innovation induced by Part $D$ to be equal to -0.56 .

We performed several sensitivity analyses to assess the robustness of our results. First, our conclusions were very similar if we used negative binomial regression. Second, our conclusions were similar if we focused only on the first FDA-approved indication for each oral chemotherapy agent. We found a $2.3 \%(95 \% \mathrm{Cl}: 0.1,4.5)$ increase in the rate of first approvals and a $7.9 \%(95 \% \mathrm{Cl}: 6.0,9.9)$ decrease in the relative survival benefits provided by these therapies after Part $D$ was implemented for every $1 \%$ increase in a cancer site's exposure to the Medicare market. Third, our findings were also similar if we excluded individual cancers or therapies from the analyses. For example, after excluding all lung cancer approvals we found a $5.5 \%(95 \% \mathrm{Cl}: 1.9,9.3)$ increase in the approval rate and $7.7 \%(95 \% \mathrm{Cl}: 5.8,9.7)$ relative decrease in the survival benefits of newer oral chemotherapy agents for every $1 \%$ increase in exposure to the Medicare market. 


\section{Infused Chemotherapy Falsification Test}

As hypothesized, we did not find evidence that the rate infused chemotherapy agents were approved before versus after Part D implementation was associated with the level of exposure to the Medicare market (Table 1; Figure 2b). We also did not find that the improvement in survival provided by newly approved infused chemotherapy agents was associated with the level of exposure to the Medicare market ( $p=0.5$, Table 2; Figure $3 b$ ).

\section{Discussion}

The creation of Medicare Part D was the largest expansion of prescription drug insurance coverage in US history. We found that the Part D expansion was importantly associated with an increase in the number of oral anti-cancer drugs approved by the FDA; however, these drugs produced smaller improvements in survival over existing therapies than their predecessors. Thus our findings suggest that there are diminishing returns to incentives for pharmaceutical innovation induced by broad coverage mandates and that more nuanced coverage and reimbursement policies may help maximize the health benefits provided by future pharmaceutical innovations.

Prior research has documented a robust response by the pharmaceutical industry to changes in market size and insurance coverage. Acemoglu and Linn (2004) found that larger markets driven by changes in US demographic trends were significantly associated with an increase in the entry of new drugs. Our finding that increases in the annual incidence of different cancer types over time were significantly associated with increases in the number of chemotherapy approvals parallel these results. Blume-Kohout and Sood (2013) found that implementation of Part D was associated with significant increases in industry-sponsored clinical trials for drug classes most likely to be affected by Medicare Part D. Our findings of substantial increases in the approval of oral anti-cancer drugs corroborate their work and add an important evaluation of the health benefits provided by the drugs eventually brought to market. 
The coverage mandates in Part D are not linked to clinical benefits, and there are no pricing constraints in this market. Thus the mandates to include "all or substantially all" anti-cancer medications on Part D formularies severely constrain health insurers' ability to negotiate on the basis of a drug's clinical or economic value by eliminating the threat of excluding the drug from the formulary (Ramsey et al. 2016). The ability to restrict the use of a product if it does not represent good value can serve as a powerful tool to lower drug prices. In 2012, Sanofi reduced the price of Zaltrap in half after Memorial Sloan Kettering Cancer Center publicly refused to use the drug, arguing that it was twice as expensive and no more effective than a similar medicine, Avastin (Pollack 2012). More recently, valuebased formularies have been tested as a way to improve value within a health care system (Yeung et al. 2016). An often-touted benefit of increased innovation - especially of duplicative efforts to develop "me too" drugs - is that the resulting competition works to lower drug prices; however, we have previously found that there is currently little pressure to lower prices in the oral anti-cancer drug market, even in the presence of increased competition (Bennette et al. 2016). This may be justified if these drugs produce benefits for different subgroups of patients; however, little evidence exists that this is the case. Thus, Part D coverage mandates coupled with high prices provide strong incentives for pharmaceutical firms to continue developing oral anti-cancer drugs even if the specific market is relatively saturated and they offer few benefits over existing therapies (Bach 2014).

Importantly, our findings of diminishing returns to health from increased pharmaceutical innovation apply only due to expanded prescription drug coverage under Medicare. The results do not apply to average or marginal investments made by the pharmaceutical industry in response to basic science advances or clinical opportunities. Recent advances in molecular and genomic science have fueled tremendous progress in anti-cancer therapies. Investments from pharmaceutical firms to develop new drugs that leverage these advances may - and if successful, likely will - return substantial health benefits to society. But many of these new treatments are also likely to be extremely 
expensive, putting even more pressure on policymakers and health insurers to ensure affordable access for patients.

How can we as a society ensure patients' access to anti-cancer drugs? Coverage mandates like those included in Medicare Part D do help protect patients' access, but they distort the market and create incentives for innovation that may heighten the underlying issue of anti-cancer drug prices rising faster than inflation and the health benefits they provide in the long run (Howard et al. 2015; Bennette et al. 2016). More nuanced coverage mandates that are linked to a drug's value or an incremental clinical benefit, might help protect patients' access to the most valuable or effective therapies while leaving insurers some leverage in negotiations with manufacturers (Bach and Pearson 2015). Another policy option, value-based pricing, would financially reward pharmaceutical firms in a manner that was proportional to the clinical benefits their drugs provide patients, and could therefore provide a powerful additional incentive for firms to invest in developing treatments that have the greatest potential health benefits for patients. We believe that accelerating efforts to develop and implement a transparent coverage and reimbursement system that is linked to the clinical benefits a drug provides would thus help improve current and future patients' access to the most effective therapies.

Our study has several limitations. First, we estimated health benefits using the improvement in median survival per drug. Although there are challenges in using median survival to compare clinical benefits of treatments across different cancers, it is reasonable for these analyses because we evaluate incremental benefits within cancers. Indeed, median survival is the most commonly reported metric of absolute health benefit in the clinical trials supporting FDA approval for the drugs in this analysis. That said, median survival measures do not incorporate potential impacts on quality-of-life and may be inadequate in capturing the health benefits of drugs that offer a small percentage of patients the chance of long term benefits. Second, we did not include estimates of survival benefits for drugs approved on the basis of surrogate endpoints or single arm studies. However, we do not expect this limitation to bias our results, as we found a very similar relationship between the rate drugs were approved and exposure to the Medicare market 
among only approvals based on survival benefits (4.2\% versus $4.6 \%)$. Lastly, there may be other factors, such as scientific advances or federal research investments, that were differential across cancers and that could have confounded the relationships we observed if they were also correlated with the proportion of newly diagnosed individuals with a specific cancer that were eligible for Medicare. Importantly, we did not observe similar trends in the market for infused chemotherapy, which we expect would be similarly impacted by most scientific advances (e.g. discovery of a new molecular target) that may have differed across cancer sites over time. Moreover, we observed a stronger association over time after (but not before) Part D was implemented for oral agents, which further suggests that the effects we observed are causal.

In conclusion, we found diminishing returns to health from increased incentives for pharmaceutical innovation created by Medicare Part D's coverage mandates for oral chemotherapy. Alternative coverage policies could create more targeted incentives for pharmaceutical research and development investments and ultimately help maximize the health benefits gained from future innovations. 
References

Acemoglu D, Linn J. Market size in innovation: theory and evidence from the pharmaceutical industry. The Quarterly Journal of Economics. August 2004:41.

Bach P. Could High Drug Prices Be Bad For Innovation? Forbes 2014.

Bach PB, Pearson SD. Payer and Policy Maker Steps to Support Value-Based Pricing for Drugs. JAMA. Dec 15 2015;314(23):2503-2504.

Bach PB. Limits on Medicare's ability to control rising spending on cancer drugs. The New England Journal of Medicine. Feb 5 2009;360(6):626-633.

Bennette CS, Richards C, Sullivan SD, Ramsey SD. Steady Increase In Prices For Oral Anticancer Drugs After Market Launch Suggests A Lack Of Competitive Pressure. Health affairs. May 1 2016;35(5):805-812.

Blume-Kohout ME, Sood N. Market Size and Innovation: Effects of Medicare Part D on Pharmaceutical Research and Development. Journal of Public Economics. Jan 2013;97:327-336.

Bowman J, Rousseau A, Silk D, Harrison C. Access to cancer drugs in Medicare Part D: Formulary placement and beneficiary cost sharing in 2006. Health Affairs 2006; 25(5): 1240-1248.

CMS proposes to test new Medicare Part B prescription drug models to improve quality of care and deliver better value for Medicare beneficiaries. 2016; https://http://www.cms.gov/Newsroom/MediaReleaseDatabase/Fact-sheets/2016Fact-sheets-items/2016-03-08.html. Accessed April 6, 2016.

DiMasi JA, Faden LB. Competitiveness in follow-on drug R\&D: a race or imitation? Nature Reviews Drug Discovery 2011; 10: 23-27.

Downing NS, Aminawung JA, Shah ND, Krumholz HM, Ross JS. Clinical trial evidence supporting FDA approval of novel therapeutic agents, 2005-2012. JAMA. Jan 22-29 2014;311(4):368-377.

Friedman JN. The Incidence of the Medicare Prescription Drug Benefit: Using Asset Prices to Assess its Impact on Drug Makers. Harvard University; 2009. Job Market Paper.

Gold LS, Buist DS, Loggers ET, et al. Advanced diagnostic breast cancer imaging: variation and patterns of care in Washington state. J Oncol Pract. Sep 2013;9(5):e194-202.

HHS Pharmaceutical Forum: Innovation, Access, Affordability and Better Health. http://www.hhs.gov/hhs-pharmaceutical-forum/. Accessed May 21, 2016.

Howard DH, Bach PB, Berndt ER, Conti RM. Pricing in the Market for Anti-cancer Drugs. Journal of Economic Perspectives. 2015;29(1):139-162.

Institute of Medicine. Delivering Affordable Cancer Care in the 21st Century: Workshop Summary. Washington, DC: The National Academies Press, 2013. doi:10.17226/18273.

Institute of Medicine. Ensuring Patient Access to Affordable Cancer Drugs: Workshop Summary. Washington, DC: The National Academies Press, 2014. doi:10.17226/18956.

Kim C, Prasad V. Cancer Drugs Approved on the Basis of a Surrogate End Point and Subsequent Overall Survival: An Analysis of 5 Years of US Food and Drug Administration Approvals. JAMA Internal Medicine. Dec 2015;175(12):1992-1994.

Lakdawalla D, Sood N, Gu Q. Pharmaceutical advertising and Medicare Part D. Journal of Health Economics. Dec 2013;32(6):1356-1367. 
Loftus P. New Push Ties Cost of Drugs to How Well They Work. Wall Street Journal2015. Mailankody S, Prasad V. Implications of Proposed Medicare Reforms to Counteract High Cancer Drug Prices. JAMA. May 52016.

Martell RE, Sermer D, Getz K, Kaitin KI. Oncology drug development and approval of systemic anti-cancer therapy by the U.S. Food and Drug Administration. The Oncologist. 2013;18(1):104-111.

Morgan S, Kennedy J. Prescription drug accessibility and affordability in the United States and abroad. Commonwealth Fund Pub 1408, 2010; vol. 49. http://www.commonwealthfund.org/ /media/Files/Publications/lssue\%20Brief/2010/] un/1408 Morgan Prescription drug accessibility US intl ib.pdf. Accessed August 2017.

Morgan S, Lopert R, Greyson D. Toward a definition of pharmaceutical innovation. Open Medicine. 2008;2(1):e4-7.

Pollack A. Sanofi Halves Price of Cancer Drug Zaltrap After Sloan-Kettering Rejection. New York Times. November 8, 2012.

Prasad V, Jena AB. Prespecified falsification end points: can they validate true observational associations? JAMA Jan 16 2013;309(3):241-242.

Prasad V, Kim C, Burotto M, Vandross A. The Strength of Association Between Surrogate End Points and Survival in Oncology: A Systematic Review of Trial-Level Meta-analyses. JAMA Internal Medicine. Aug 2015;175(8):1389-1398.

Ramsey SD, Lyman GH, Bangs R. Addressing Skyrocketing Cancer Drug Prices Comes With Tradeoffs: Pick Your Poison. JAMA Oncology. Apr 1 2016;2(4):425-426.

Scherer FM. The link between gross profitability and pharmaceutical R\&D spending. Health Affairs. Sep-Oct 2001; 20(5):216-220.

Yeung, K., Basu, A., Hansen, R. N., Watkins, J. B., \& Sullivan, S. D. (2016). Impact of a Valuebased Formulary on Medication Utilization, Health Services Utilization, and Expenditures. Medical Care. DOI: 10.1097/MLR.0000000000000630 
Figure 1. Relative change in number of FDA approvals in (a) 2011-September 2016 (post-Part D) compared to (b) 1994-2003 (pre-Part D) by a cancer's exposure to the Medicare market.

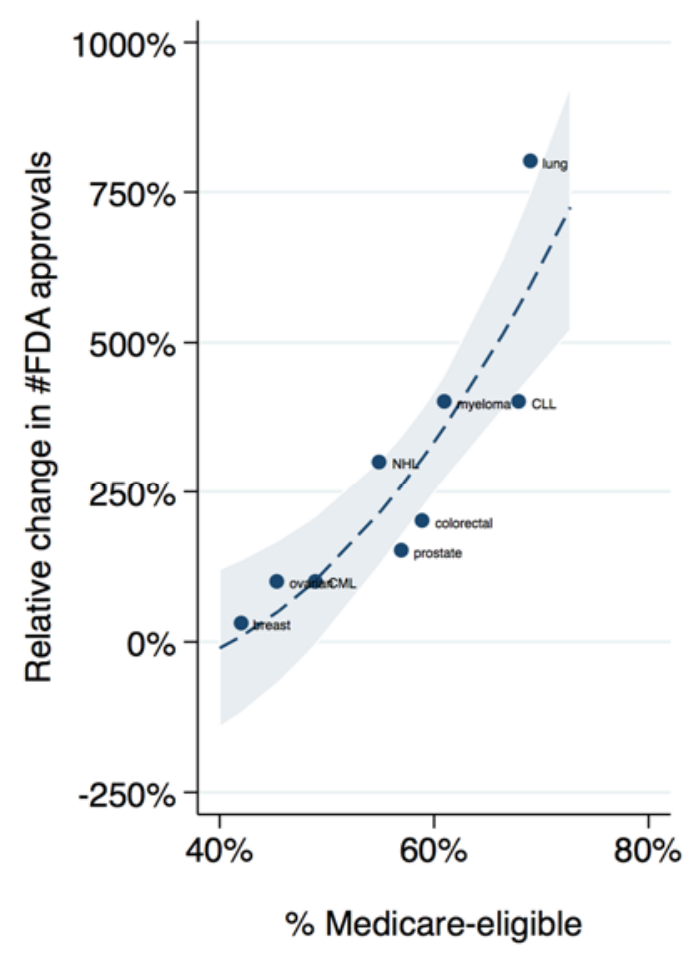

(a)

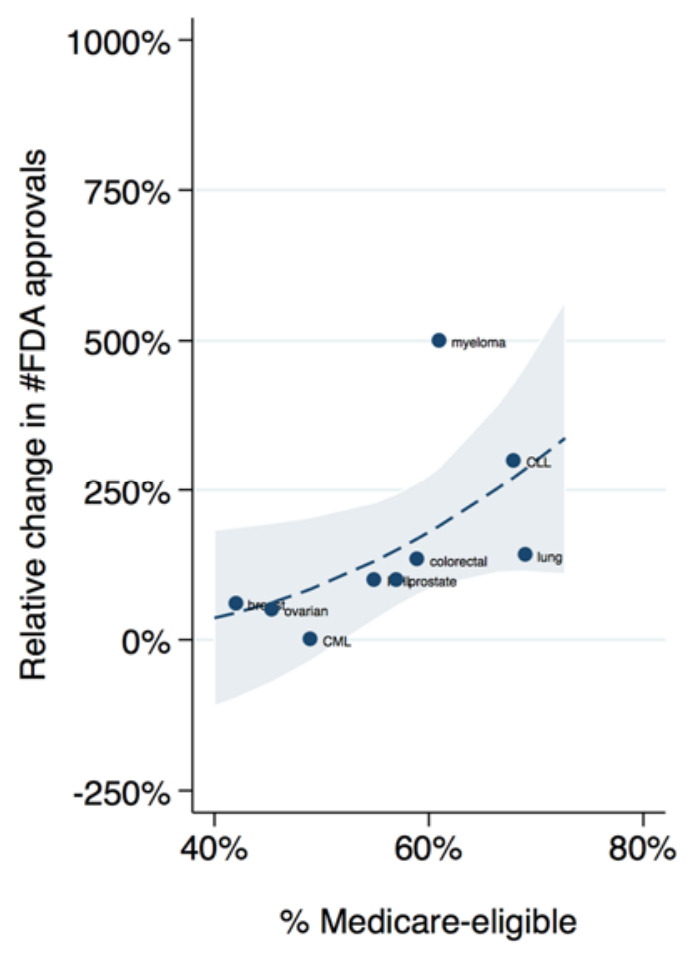

(b) 
Figure 2. Percent change in number of (a) oral anticancer medications and (b) infused chemotherapy agents approved in various time periods, relative to number approved 19941998 , for every $1 \%$ increase in a cancer site's exposure to the Medicare market. (NOTE: Estimates are from Poisson regression models with robust standard errors and adjusted for changes in the annual incidence of the cancer site over time.)

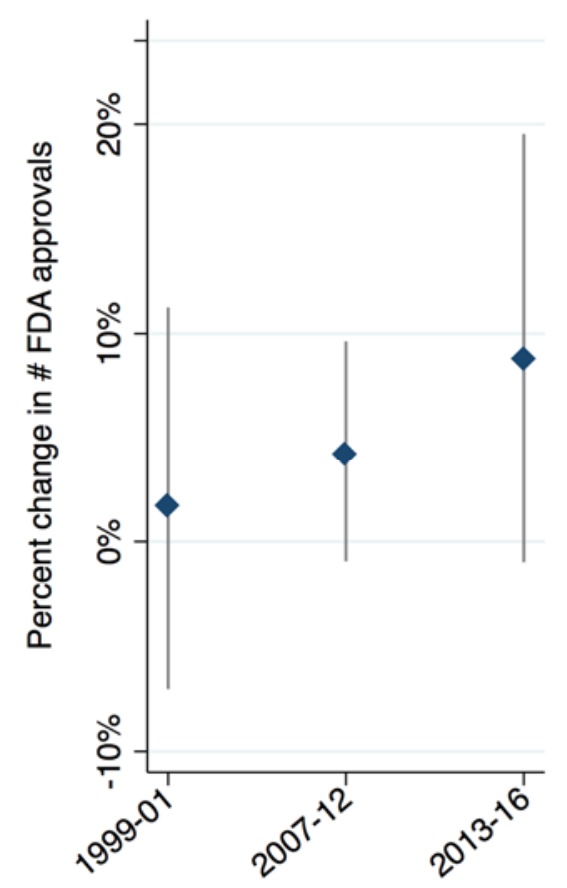

(a)

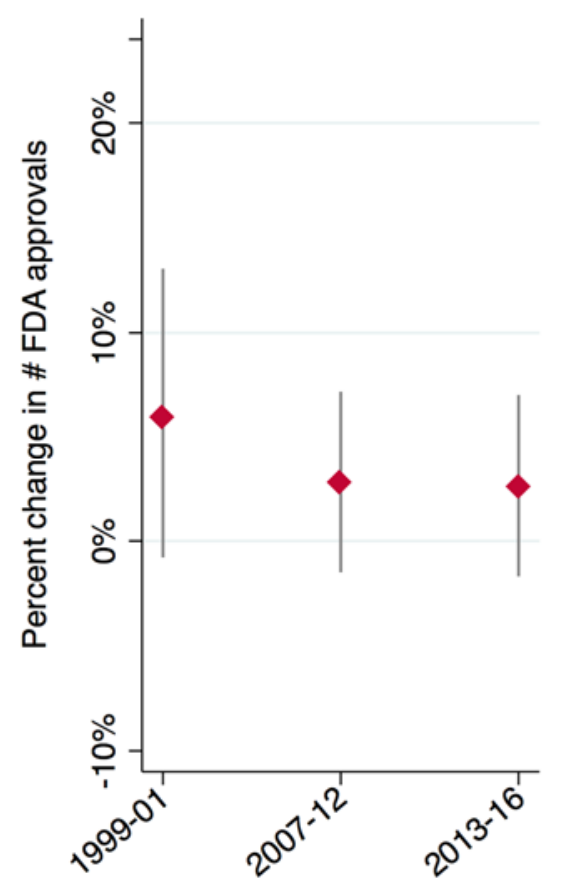

(b) 
Figure 3. Percent change in the survival benefits from (a) oral anticancer medications and (b) infused chemotherapy agents approved to treat cancer in various time periods, relative to those approved 1994-1998, for every $1 \%$ increase in a cancer site's exposure to the Medicare market. (NOTE: All estimates are from Poisson regression models with robust standard errors and adjusted for changes in the annual incidence of the cancer over time.)

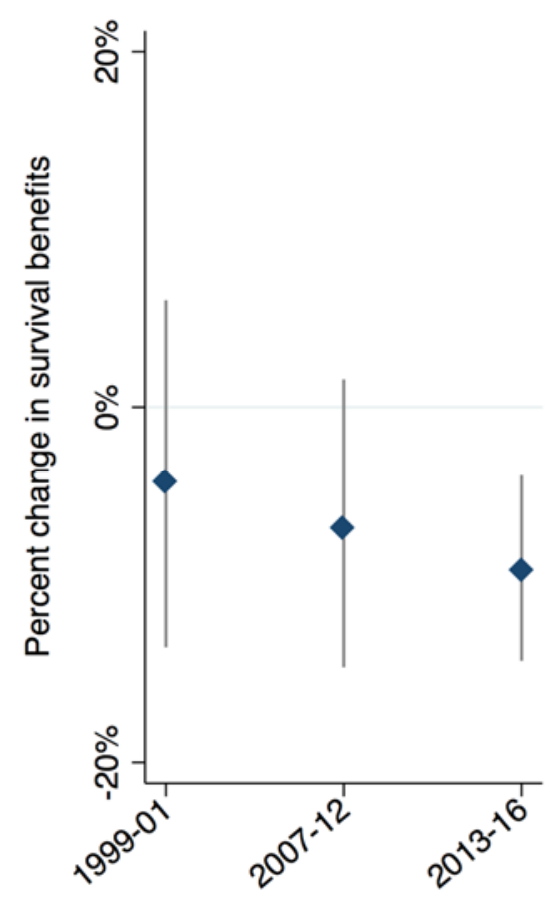

(a)

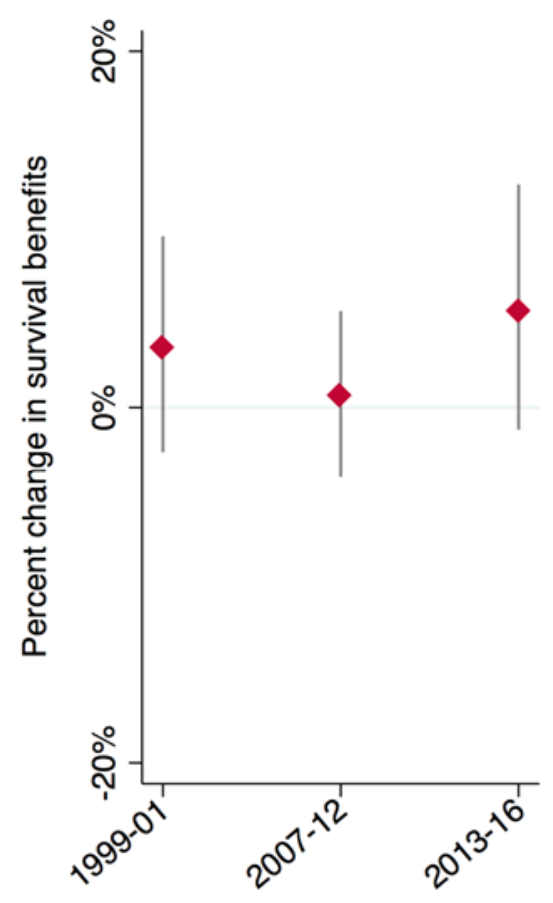

(b) 
Table 1. Relative change in the rate chemotherapy agents are approved by the FDA, in pre-Part D (1994-2003) versus post-part D (2008-2016) time period. Models included cancer and year fixed effects and a binary indicator for post-Part D period.

\begin{tabular}{lccc}
\hline Independent variable & $\begin{array}{c}\text { Relative } \\
\text { change }\end{array}$ & $\mathbf{9 5 \%} \mathbf{C l}$ & $\boldsymbol{p}$ value \\
\hline $\begin{array}{l}\text { Oral chemotherapy } \\
\quad \text { \% increase in exposure to Medicare } \\
\text { market }\end{array}$ & 1.057 & $1.017,1.098$ & 0.005 \\
$\begin{array}{l}\text { 1\% increase in annual incidence } \\
\text { Infused chemotherapy } \\
\quad \text { 1\% increase in exposure to Medicare } \\
\text { market } \\
1 \% \text { increase in annual incidence }\end{array}$ & 1.406 & $1.111,1.781$ & 0.005 \\
\end{tabular}


Table 2. Relative change in absolute survival benefits reported in drug's label in pre-Part D (19942003) versus post-part D (2008-2016) time period. Models included cancer and year fixed effects and a binary indicator for post-Part $D$ period.

\begin{tabular}{lccc}
\hline Independent variable & $\begin{array}{c}\text { Relative } \\
\text { change }\end{array}$ & $\mathbf{9 5 \%} \mathbf{C l}$ & $\boldsymbol{p}$ value \\
\hline Oral chemotherapy: & & & $<0.001$ \\
1\% increase in exposure to Medicare market & 0.968 & $0.957,0.979$ & 0.013 \\
1\% increase in annual incidence & 0.810 & $0.686,0.957$ & \\
Infused chemotherapy: & & & 0.5 \\
1\% increase in exposure to Medicare market & 1.018 & $0.969,1.069$ & 0.2 \\
\hline 1\% increase in annual incidence & 1.317 & $0.875,1.983$ & \\
\hline
\end{tabular}

